\title{
STOPPING POWER, ITS MEANING, AND ITS GENERAL CHARACTERISTICS*
}

\author{
Mitio Inokuti \\ Argonne National Laboratory, Argonne, IL 60439
}

\begin{abstract}
This essay presents remarks on the meaning of stopping power and of its magnitude. More precisely, the first set of remarks concerns the connection of stopping power with elements of particle-transport theory, which describes particle transport and its consequences in full detail, including its stochastic aspects. The second set of remarks concerns the magnitude of the stopping power of a material and its relation with the material's electronic structure and other properties.
\end{abstract}

\section{DISCLAIMER}

This report was prepared as an account of work sponsored by an agency of the United States Government. Neither the United States Government nor any agency thereof, nor any of their employees, makes any warranty, express or implied, or assumes any legal liability or responsibility for the accuracy, completeness, or usefulness of any information, apparatus, product, or process disclosed, or represents that its use would not infringe privately owned rights. Reference herein to any specific commercial product, process, or service by trade name, trademark, manufacturer, or otherwise does not necessarily constitute or imply its endorsement, recommendation, or favoring by the United States Government or any agency thereof. The views and opinions of authors expressed herein do not necessarily state or reflect those of the United States Government or any agency thereof.

The submitted manuseript has been authored
by a contractor of the U. S. Government
under contract No. W-31-109ENG-38.
Accordingly, the U.S. Government retains a
nonexclusive, soyalty-free license to publish
or reproduce the published form of this
contribution, or allow others to do so, for
U.S. Government purposes.

* Work supported in part by the U.S. Department of Energy, Office of Energy Research, Office of Health and Environmental Research, under Contract W-31-109-Eng-38. 


\section{DISCLAIMER}

Portions of this document may be illegible in electronic image products. Images are produced from the best available original document. 


\section{What Do We Mean by Stopping Power?}

An experimentalist naturally thinks of the stopping power as the mean energy loss of an energetic particle per unit path length in a given material. Suppose that a particle of given kinetic energy $\mathrm{T}$ passes through a thickness $\Delta \mathrm{x}$ and emerges with kinetic energy $\mathrm{T}$. Repeated trials will result in a distribution of $T^{\prime}$ values. If the mean of $T-T^{\prime}$ is $\Delta T$, the stopping power is the limit of $\Delta \mathrm{T} / \Delta \mathrm{x}$ as $\Delta \mathrm{x} \rightarrow 0$. The limit $\Delta \mathrm{x} \rightarrow 0$ is an idealization; in practice it means that the path length is sufficiently short. We should ask what is meant by "sufficiently short." We should also recognize that the above definition presumes that a particle travels virtually along a straight line, without appreciable deflection. This picture indeed applies to any particle of very high energy, but it becomes progressively more unrealistic with decreasing energy, especially for a light particle such as an electron or a positron.

A theoretician usually evaluates the stopping power from the cross section for a single collision. Consider a material consisting of n structural units per unit volume. For brevity, let us call the structural unit a molecule. Suppose that $[d \sigma(T, E) / d E] d E$ represents the cross section for a collision of a particle of kinetic energy $T$ with a molecule, resulting in energy transfer between $\mathrm{E}$ and $\mathrm{E}+\mathrm{dE}$. Then, the stopping power is evaluated as $\mathrm{n} \int \mathrm{dE} \mathrm{E} d \sigma(\mathrm{T}, \mathrm{E}) / \mathrm{dE}$.

Are the definitions of the experimentalist and the theoretician equivalent? The answer is certainly yes, if the material is so thin that a particle collides with a molecule only once at most. Indeed, cross-section measurements are carried out for this circumstance, and then a distribution of energy losses is called the energy-loss spectrum. However, when the material is thick enough to cause multiple collisions, the question warrants some discussion.

\section{Elementary Treatment of Multiple Collisions}

For convenience, we define the normalized collision spectrum 


$$
\pi(T, E)=\frac{d \sigma(T, E)}{d E} / \int_{0}^{E_{\max }} d E \frac{d \sigma(T, E)}{d E}
$$

where $d \sigma(T, E) / d E$ is the (differential) cross section for energy transfer, as discussed earlier. The quantity $\pi(T, E) d E$ signifies the probability that a particle of kinetic energy $T$ transfers energy ranging from $E$ to $E+d E$ upon a collision with a molecule. The upper limit $E_{\max }$ of the integration depends on $T$. When $T$ is so high that the Bethe theory applies [1], we may take

$$
E_{\max }=2 m v^{2} /\left(1-\beta^{2}\right),
$$

where $v=\beta c$ is the particle speed, $c$ is the speed of light in a vacuum, and $m$ is the electron mass. At relativistic speeds, $E_{\max }$ depends on $T$ very weakly. The dependence of $E_{\max }$ on $T$ is also moderate at lower speeds, except near the thresholds of electronic excitation of the material.

Let us assume that the cross section $d \sigma(T, E) / d E$ is a product of a function $Q(T)$ of $T$ only and a function $q(E)$ of $E$ only. This is true for the Rutherford cross section and approximately so for the Bethe cross section [1]. Let us also restrict our consideration to a modest number $\mathrm{k}$ of multiple collisions, so that the total energy loss in these collisions is much smaller than the particle energy $T$. Under these conditions we may regard $\pi(T, E)$ as nearly independent of $T$ and write it as $p(E)$, a function of $E$ alone.

Suppose that $p_{k}(E) d E$ is the probability that $k$ collisions result in a total energy loss ranging from $E$ to $E+d E$. We assume that the successive collisions are independent. (In the language of the probability theory, we regard multiple collisions as a stationary Markovian process [2].) Then the probability $\mathrm{p}_{\mathrm{k}+1}(\mathrm{E})$ of the total energy loss $\mathrm{E}$ after $\mathrm{k}+1$ collisions is the product of the probability $\mathrm{p}_{\mathrm{k}}(\varepsilon)$ of energy loss $\varepsilon$ after $\mathrm{k}$ collisions and the probability $p(E-\varepsilon)$ of energy loss $E-\varepsilon$, summed over all possible values of $\varepsilon$. That is to say, 


$$
p_{k+1}(E)=\int_{0}^{E_{\max }} d \varepsilon p_{k}(\varepsilon) p(E-\varepsilon)
$$

for any integer $k=1,2,3, \ldots$, with the stipulation $p_{1}(E)=p(E)$.

The mean energy loss after $k$ collisions

$$
\overline{\mathrm{E}}_{\mathrm{k}}=\int_{0}^{\mathrm{kE} \mathrm{max}_{\max }} \mathrm{dEE}_{\mathrm{k}}(\mathrm{E})
$$

is $k$ times the mean energy loss $\bar{E}$ for a single collision. That is,

$$
\overline{\mathrm{E}}_{\mathrm{k}}=\mathrm{k} \overline{\mathrm{E}}
$$

where

$$
\overline{\mathrm{E}}=\int_{0}^{\mathrm{E}_{\max }} \mathrm{dEEp}(\mathrm{E})
$$

The proof of Eq. (5) is elementary; it just requires showing that

$$
\overline{\mathrm{E}}_{\mathrm{k}+1}=\overline{\mathrm{E}}_{\mathrm{k}}+\overline{\mathrm{E}}
$$

which follows from Eqs. (3) and (4) through a change of variables in repeated integrations. Equation (7) leads to Eq. (5). See Fig. 1 and its caption for details.

The precise number $\mathrm{k}$ of collisions occurring during a given path length is not uniquely fixed but is stochastically distributed. If the probability of each collision is vanishingly small, we may assume Poisson statistics. Let $\mathrm{P}(\mathrm{E}, \mu) \mathrm{dE}$ be the probability that a particle loses energies ranging from $E$ to $E+d E$ in a path length in which the mean number of collisions is $\mu$. Then, we may write 


$$
P(E, \mu)=e^{-\mu} \sum_{k=0}^{\infty} \frac{\mu^{k}}{k !} p_{k}(E)
$$

This function, called the straggling function, has been the object of various studies [3]. The mean energy loss with respect to $P(E, \mu)$,

$$
\bar{E}(\mu)=\int_{0}^{\infty} \mathrm{dE} \mathrm{EP}(\mathrm{E}, \mu)
$$

is readily calculable with the use of Eq. (5). The result is

$$
\overline{\mathrm{E}}(\mu)=\mathrm{e}^{-\mu} \sum_{\mathrm{k}=0}^{\infty} \frac{\mu^{\mathrm{k}}}{\mathrm{k} !} \mathrm{k} \overline{\mathrm{E}}=\mu \overline{\mathrm{E}} .
$$

To paraphrase, the mean energy loss $\bar{E}$ is proportional to the mean collision number $\mu$ and thence to the path length. The constant of proportionality is $\overline{\mathrm{E}}$, the mean energy loss in a single collision. Thus, we see one way to relate the experimentalist's definition with the theoretician's definition of stopping power. This conclusion rests on a number of assumptions, including the following. First, the path length considered must be short enough to justify the use of $p(E)$, which is independent of the current energy of the particle. Yet, in an experiment, the path length needs to be long enough to lead to clearly measurable energy losses. Second, the particle deflection should be negligible. This is true at high energies, but it becomes uncertain at low energies, especially for electrons or positrons. Consequences of the failure of these two assumptions were discussed by Sigmund [4]. Third, successive collisions should be independent. This condition is highly plausible at high energies and in a medium of low density, but it becomes questionable at low energies, especially in a highdensity material. Finally, we tacitly assumed that the material is spatially homogeneous. In practice, a material used in an experiment may contain some kind of inhomogeneity, such as domains of different atomic arrangements and boundaries between them. An extreme example is biological material, which as a rule involves structures of successive orders of 
scale.

\section{Connections with Particle Transport Theory}

\subsection{The Landau Equation}

Transport theory is one way of describing particle penetration and energy loss in detail (specifically including stochastic aspects). Let us consider energetic heavy particles penetrating a material along a straight line. We assume that $\mathrm{u}(\mathrm{T}, \mathrm{s}) \mathrm{dT}$ source particles with kinetic energies ranging from $T$ to $T+d T$ are steadily introduced at position $s$. Let $f(T, s) d T$ be the number of particles with kinetic energies of $T$ to $T+d T$ that are found at position $s$. Then, $f(T, s)$ obeys the equation, originally derived by Landau [5],

$$
\partial f(T, s) / \partial s=K f(T, s)+u(T, s)
$$

where $\mathrm{K}$ is an operator defined by

$$
\begin{aligned}
K f(T, s) & =n \int_{0}^{E_{\max }(T+E)} d E \frac{d \sigma(T+E, E)}{d E} f(T+E, s) \\
& -n \int_{0}^{E_{\max }(T)} d E \frac{d \sigma(T, E)}{d E} f(T, s) .
\end{aligned}
$$

The first term on the right-hand side of Eq. (12) represents the number of particles that had energy $T+E$, lost energy $E$ in a collision, and therefore now have energy $T$. The second term represents the number of particles, present at $s$ with energy $T$, that lose energy $E$ in a collision. Thus, $\operatorname{Kf}(T, s)$ represents the net change in the number of particles at energy $T$ and at position $\mathrm{s}$ due to collisions, integrated over all possible values of energy loss $\mathrm{E}$.

The formal solution of the integral equation, Eq. (11), is possible by use of the Laplace transform, as discussed by Fano [6]. Here we focus on the connection of Eq. (11) with the stopping power. For this purpose, we make two assumptions. First, we assume that every 
energy loss $E$ is much smaller than $T$. Second, we disregard the dependence on $T$ of the upper limit $E_{\max }$ of the $E$ integration. Then it is possible to simplify the analytic structure of the $\mathrm{K}$ operator considerably. The result is

$$
\begin{aligned}
K f(T, s) & =\frac{\partial}{\partial T}\left[n \int_{0}^{E_{\max }(T)} d E E \frac{d \sigma(T, E)}{d E} f(T, s)\right] \\
& -n \frac{d E_{\max }(T)}{d T} E_{\max }(T) \frac{d \sigma\left(T, E_{\max }(T)\right)}{d E} f(T, s) .
\end{aligned}
$$

Again, we recall the weak dependence of $E_{\max }(T)$ on $T$ and drop the second term to obtain

$$
K f(T, s)=\frac{\partial}{\partial T}[S(T) f(T, s)]
$$

where $S(T)$ is the stopping power, defined as

$$
S(T)=n \int_{0}^{E_{\max }(T)} d E E \frac{d \sigma(T, E)}{d E} .
$$

Then, the Landau equation, Eq. (11), takes the form of a first-order partial differential equation

$$
\frac{\partial f(T, s)}{\partial s}=\frac{\partial}{\partial T}[S(T) f(T, s)]+u(T, s)
$$

Multiplying the both sides by $S(T)$, we see that the product $S(T) f(T, s)$ is a suitable dependent variable. Namely, we write

$$
\frac{\partial}{\partial s}[S(T) f(T, s)]=S(T) \frac{\partial}{\partial T}[S(T) f(T, s)]+S(T) v(T, s)
$$

Next we introduce an independent variable $x$ of the length dimension such that 


$$
-\frac{\mathrm{d}}{\mathrm{dx}}=\mathrm{S}(\mathrm{T}) \frac{\mathrm{d}}{\mathrm{dT}}, \quad \text { or } \quad \frac{\mathrm{dx}}{\mathrm{dT}}=-\frac{1}{\mathrm{~S}(\mathrm{~T})}
$$

Then, we may recast Eq. (17) in a compact form

$$
\left(\frac{\partial}{\partial s}+\frac{\partial}{\partial x}\right) S(T) f(T, s)=S(T) u(T, s)
$$

The solution of this equation can be obtained by an elementary analytic procedure, as fully discussed by Inokuti et al. [7]. The key point of the procedure is to see that a homogeneous equation

$$
\left(\frac{\partial}{\partial s}+\frac{\partial}{\partial x}\right) g(s, x)=0
$$

has the general solution $\mathrm{g}(\mathrm{s}, \mathrm{x})=\chi(\mathrm{s}-\mathrm{x})$, where $\chi$ is a differentiable function to be determined by an initial condition.

The meaning of the variable $x$ is apparent from Eq. (18): the increment $d x$ accompanying the infinitesimal decrease $-\mathrm{dT}$ is $-\mathrm{dT} / \mathrm{S}(\mathrm{T})$. In other words,

$$
x=\int_{T}^{T_{0}} \frac{d T}{S\left(T^{*}\right)}
$$

represents the position of a hypothetical particle that started at the origin with energy $\mathrm{T}_{0}$ and now has a lower energy $T$. Such a particle is hypothetical (1) because a real particle loses energy in a variety of ways described by the cross section $d \sigma(T, E) / d E$ in the statistical sense and (2) because we made the assumptions to simplify the $\mathrm{K}$ operator. It is customary to call $x$ the CSDA (continuous slowing-down approximation) path length. 
Another remark concerns the product $\mathrm{S}(\mathrm{T}) \mathrm{f}(\mathrm{T}, \mathrm{s})$, which naturally emerges as the dependent variable (i.e., an appropriate object of consideration). For a monoenergetic, localized source $u(T, s)=\delta\left(T-T_{0}\right) \delta\left(s-s_{0}\right)$, where $T_{0}$ and $s_{0}$ are constant, the solution of Eq. (19) is in effect that of a homogeneous equation, Eq. (20). Therefore, the product $S(T) f(T, s)$ practically retains the value given by the initial condition. In other words, $f(T, s)$ is close to the reciprocal of $\mathrm{S}(\mathrm{T})$. Roughly speaking, $1 / \mathrm{S}(\mathrm{T})$ is a zero-order approximation to $f(T, s)$ where the CSDA is sensible.

\subsection{The Track-Length Distribution}

The quantity

$$
y(T)=\int_{0}^{\infty} d s f(T, s)
$$

is of particular interest in its connection with the stopping power. Its physical meaning is as follows: $y(T) d T$ represents the path length traveled by each particle while its kinetic energy is between $T$ and $T+d T$. Thus, $y(T)$ is called the track-length distribution.

The significance of $y(T)$ lies in its role in the evaluation of the yield of a product generated as a result of the penetration of particle a into matter. To paraphrase, we may wish to calculate the total number of ions generated in matter through the complete slowing down of an energetic particle, for instance, as a basis for dosimetry with the use of an ionization chamber. For a full discussion on this topic, see Inokuti et al. [8]. The total number of product $\alpha$ generated is given by $n \int \mathrm{dT} y(\mathrm{~T}) \sigma_{\alpha}(\mathrm{T})$, where $\sigma_{\alpha}(\mathrm{T})$ is the cross section for the generation of $\alpha$ in a single collision of a particle at energy $T$. This statement readily follows from the well-known definition of the cross section. Note in particular its dimensions: the number density $n$ of the medium molecules has the dimension of (length) ${ }^{-3}$, $y(T) d T$ has the dimension of length, and $\sigma_{\alpha}(T)$ has the dimension of (length) ${ }^{2}$. 
The track-length distribution obeys the equation

$$
K y(T)+U(T)=0
$$

where

$$
U(T)=\int_{0}^{\infty} \mathrm{ds} u(T, s)
$$

is the spatially integrated source spectrum, as we can readily see from Eqs. (11) and (22). We may call Eq. (23) the Fano equation, in view of the initial treatment of heavy particles [9]. However, the treatment of electrons [10] is now better known, and therefore the term "Spencer-Fano equation" is more generally recognized.

In the CSDA, i.e., within the set of assumptions leading to the simplification of the $\mathrm{K}$ operator, we can write

$$
\frac{d}{d T}[S(T) y(T)]+U(T)=0
$$

which can be solved as

$$
s(T) y(T)=\int_{T}^{T_{0}} d T^{\prime} U\left(T^{\prime}\right)
$$

Here the product $S(T) y(T)$ is readily calculable from the source spectrum $U(T)$ of the particles. In other words, the nontrivial part of $y(T)$ is the reciprocal of the stopping power $S(T)$. To paraphrase further, $[S(T)]^{-1}$ is the zeroth-order approximation to $y(T)$, which characterizes the track-length distribution fully, including the stochastic aspects of particle penetration. 
In conclusion, we have seen that knowledge of the stopping power alone may have limited significance, but it is an indispensable basis for transport theory.

\section{Reflections on the Assumption of the Markov Process for Successive Collisions}

In a medium with a modest density of molecules (e.g., in a gas), it is reasonable to assume that successive collisions of a fast charged particle are statistically independent. In condensed matter, however, the successive collisions may not be independent, especially for particles of low speeds and low masses. The following are examples of circumstances in which the assumption of independent collisions is questionable.

First, as pointed out at the end of Section 2, any spatial inhomogeneity of a material is an obvious example. Second, a striking example is the channeling of particles in a crystal; a particle incident within a small angle with respect to atomic layers glances many atoms only at large impact parameters and thus suffers from substantially smaller energy losses throughout its passage through the crystal than would be expected from the ordinary stopping power $[11,12]$. Third, an ion resulting from a collision of a particle produces an electric field that affects nearby molecules, and therefore the probability $p(E)$ of energy transfer $E$ in the next collision may differ appreciably from that in the first collision. This effect should be present whenever the stopping power is large, especially, for highly charged particles. However, I am unaware of either a serious theoretical analysis of this effect or an unambiguous experimental finding pertinent to it. Finally, for electrons or positrons of low kinetic energies traveling in condensed matter, wave-mechanical effects need to be considered. Recall that the de Broglie wavelength $\lambda$ of an electron or positron of kinetic energy $\mathrm{T}$ (in units of $\mathrm{eV}$ ) is $(150 / \mathrm{T})^{1 / 2} \times 10^{-8} \mathrm{~cm}$. Therefore, $\lambda$ is comparable to interatomic or intermolecular distances in ordinary condensed matter, for $\mathrm{T}$ below a few hundred $\mathrm{eV}$, and is greater for lower T. Consequently, an electron or positron at such low energies may behave in a way hardly compatible with a picture of independent successive collisions. 


\section{The Magnitude of the Stopping Power}

Let us now turn to the magnitude of the stopping power, more specifically of the stopping cross section

$$
\sigma_{\mathrm{st}}(T)=\int \mathrm{dEE} \frac{\mathrm{d} \sigma(T, E)}{\mathrm{dE}}
$$

for a molecule (or an atom or a structural unit of condensed matter). As a rule of thumb, the two factors in the integrand, $\mathrm{E}$ and $\mathrm{d} \sigma(\mathrm{T}, \mathrm{E}) / \mathrm{dE}$, tend to balance each other. In other words, when $E$ is small, $d \sigma(T, E) / d E$ tends to be large; when $E$ is large, $d \sigma(T, E) / d E$ tends to be small. This tendency is easily understandable as soon as we consider electrons bound with different strengths. A weakly bound electron occupies a large spatial region and therefore is affected by a collision of an external particle at high probability; however, energy transfer in the collision is likely to be modest. A tightly bound electron occupies a small spatial region and therefore is affected by a collision at low probability; however, energy transfer in the collision, if it ever occurs, is likely to be appreciable. Electrons in different shells of an atom represent a good illustration of the tendency: outer-shell electrons give rise to larger cross sections and smaller energy transfers, inner-shell electrons to smaller cross sections and larger energy transfers.

For fast charged particles, the same tendency finds a precise expression in the Bethe sum rule for the generalized oscillator strength, as discussed by Inokuti [1] and by Fano [6]. Roughly speaking, the Bethe sum rule says that the mean energy transfer to a molecule by collisions of a fast charged particle at a fixed momentum transfer (which is a technically important proviso) is independent of the electronic structure and is fixed by the total number of electrons alone.

To appreciate the general characteristics of the stopping cross section, it is heuristic to consider the total cross section for inelastic collisions, 


$$
\sigma_{\mathrm{tot}}(T)=\int d E \frac{d \sigma(T, E)}{d E}
$$

On the general grounds sketched in the opening paragraph of this section, the magnitude of $\sigma_{\text {tot }}$ should be governed by the spatial extent of atomic electrons; for instance, outer-shell electrons should contribute predominantly and inner-shell electrons modestly. In the Bethe theory, which applies to a charged particle with speeds far exceeding those of all atomic electrons, it is possible to show [1] that an atomic property crucial to the magnitude of $\sigma_{\text {tot }}$ is the ground-state expectation value $\left\langle\left(\Sigma_{j} x_{j}\right)^{2}\right\rangle$, where $x_{j}$ is the coordinate component of the $j^{\text {th }}$ atomic electron. To be precise, for a particle of charge ze and (nonrelativistic) speed $v$, we may write

$$
\sigma_{\mathrm{tot}}(\mathrm{T})=4 \pi \mathrm{z}^{2}\left(\mathrm{e}^{2} / \hbar \mathrm{v}\right)^{2}\left\langle\left(\Sigma_{\mathrm{j}} \mathrm{x}_{\mathrm{j}}\right)^{2}\right\rangle \ln \left[\left(2 \mathrm{mv} \mathrm{v}^{2} / \mathrm{R}\right) c_{\mathrm{tot}}\right]
$$

where $\mathrm{R}=\mathrm{me}^{4} /\left(2 \hbar^{2}\right)=13.6 \mathrm{eV}$ is the Rydberg energy, and $c_{\text {tot }}$ is a dimensionless quantity of the order of unity determined by the electronic structure. The logarithmic factor thus depends mildly on the electronic structure. Notice that $\mathrm{e}^{2 / \hbar}$ is the atomic unit for speed and that $\left\langle\left(\Sigma_{j} x_{j}\right)^{2}\right\rangle$ has the dimension of length squared, which is appropriate for the cross section.

An illustration of the relation of $\sigma_{\text {tot }}$ with the electronic structure of atoms is seen in Fig. 2 taken from Inokuti et al. [13], which shows $\sigma_{\text {tot }}$ of atoms with atomic numbers $Z$ up to 38 , for $50-\mathrm{keV}$ electrons. The total inelastic-collision cross section shows marked periodic variations, reflecting the shell structure, together with a mildly increasing trend with $Z$.

In sharp contrast, the stopping cross section $\sigma_{\text {tot }}(\mathrm{T})$ within the Bethe theory is roughly proportional to $Z$. To be precise, we may write [1]

$$
\sigma_{\mathrm{st}}(\mathrm{T})=8 \pi \mathrm{z}^{2}\left(\mathrm{e}^{2} / \hbar \mathrm{v}\right)^{2}\left(\mathrm{Ra}_{0}^{2}\right) \mathrm{Z} \ln \left(2 \mathrm{mv} \mathrm{v}^{2} / \mathrm{I}\right)
$$

where $I$ is the mean excitation energy, and $\mathrm{a}_{0}=\hbar^{2} / \mathrm{me}^{2}=0.529 \times 10^{-8} \mathrm{~cm}$ is the Bohr radius. 
Notice that the factor $\mathrm{Ra}_{0}^{2}$ has the dimension appropriate for the stopping cross section. Here again, the logarithmic factor depends on the electronic structure only mildly.

The balancing trend of the two factors in the integrand of Eq. (27) means that the stopping cross section per electron depends only weakly on electronic structure, if all of the electrons contribute fully to the energy losses of incident particles. This is why the effects of chemical binding in a molecule or of atomic aggregation in condensed matter are generally modest for very fast charged particles. These effects are expected to become appreciable when only some of the electrons contribute fully to the energy losses, or when the balancing trend does not apply for any other reason. A readily recognizable example of such circumstances occurs for low-energy particles that cannot effectively excite or ionize innershell electrons, and for materials that contain so many atoms of appreciable atomic numbers that a sizable fraction of atomic electrons occupy inner shells. For low-energy particles passing through matter, electron captures and losses also occur at high probabilities, invalidating the balancing trend. Indeed, the work of Arnau et al. [14], who thoroughly explained the differences in the stopping cross sections of zinc for the vapor and metallic states, is fully understandable from my point of view.

\section{A Linguistic Question}

Finally, I wish to share with the reader reflections on a question raised by a student: Isn't it strange that the stopping power has the dimension of force $=$ energy/length, rather than the dimension of power $=$ energy/time? My examination of the early literature on the subject indicates that the term "stopping power" got into the English language as the translation for the German "Bremsvermögen," which literally means the capability of braking.

The word "power" here is used in the same way as in "resolving power," familiar in microscopy and spectroscopy. The mean energy loss of a moving particle per unit time is given by $v S(T)$, where $v$ is the speed of the particle of kinetic energy $T$. If we were to adhere to a rigorous logic, $\mathrm{vS}(\mathrm{T})$ would be the quantity corresponding to the term "stopping power." 
The word "stopping" is also debatable. The verb "stop" usually implies "come to rest," while the German verb "bremsen" means "brake" or "decelerate." Thus, a better translation of Bremsvermögen would be "braking force" or "decelerating force." However, the usage of words is not always logical but is often based on the prevailing tradition. To avoid a misunderstanding, I am not proposing a change in the well-established technical term "stopping power."

After all, the student's question is stimulating as is often the case, and is also meaningful from the point of view of physics. The stopping power is indeed the force exerted by the medium on a moving particle. In technical terms; the stopping power may be viewed as the slope of the wake potential at the particle position, as first pointed out by Bohr [15] and analytically shown by Echenique et al. [16].

\section{Acknowledgments}

I thank M. A. Dillon, U. Fano, and M. Kimura for valuable comments on an earlier manuscript of this article. 


\section{$\underline{\text { References }}$}

[1] M. Inokuti, Rev. Mod. Phys. $\underline{43}$ (1971) 297.

[2] N. G. van Kampen, Stochastic Processes in Physics and Chemistry, North-Holland, Amsterdam, 1981.

[3] H. Bichsel, Rev. Mod. Phys. 60 (1988) 663.

[4] P. Sigmund, in Interaction of Charged Particles with Solids and Surfaces, A. GrasMarti, H. M. Urbassek, N. R. Arista, and F. Flores (eds.), (Plenum Press, New York, 1990) p. 73.

[5] L. Landau, J. Phys. U.S.S.R. $\underline{8}$ (1944) 201.

[6] U. Fano, Ann. Rev. Nucl. Sci. 13 (1963) 1.

[7] M. Inokuti, M. Kimura, and M. A. Dillon, Phys. Rev. A 38 (1988) 1217.

[8] M. Inokuti, M. Kimura, and M. A. Dillon, in Advances in Chemical Physics, Vol. $84_{2}$ edited by I. Prigogine and S. A. Rice, John Wiley \& Sons, 1993, p. 193.

[9] U. Fano, Phys. Rev. $\underline{92}$ (1953) 328.

[10] L. V. Spencer and U. Fano, Phys. Rev. 93 (1954) 1172.

[11] J. Lindhard, Phys. Lett. 12 (1964) 126.

[12] H. Esbensen and J. A. Golovchenko, Nucl. Phys. A298 (1978) 382.

[13] M. Inokuti, J. L. Dehmer, T. Baer, and J. D. Hanson, Phys. Rev. A 23 (1981) 95.

[14] A. Arnau, P. Bauer, F. Kastner, A. Salin, V. H. Ponce, P. D. Fainstein, and P. M. Echenique, Phase effect in the energy loss of hydrogen projectile in zinc targets, a preprint.

[15] N. Bohr, K. Dan. Vidensk. Selsk. Mat. Fys. Medd. 18 (1948) No. 8.

[16] P. M. Echenique, R. H. Ritchie, and W. Brandt, Phys. Rev. B 20 (1979) 2567. 


\section{Figure Captions}

Fig. 1. Diagram showing the procedure for the proof of Eq. (5) and thence of Eq. (7).

Inserting Eq. (3) into Eq. (4), we write $\bar{E}_{k+1}=\int_{0}^{(k+1) E_{\max }} \mathrm{dEE} \int_{0}^{\mathrm{E}} \mathrm{d} \varepsilon \mathrm{p}_{\mathrm{k}}(\varepsilon) \mathrm{p}(\mathrm{E}-\varepsilon)$.

The domain of the double integration, given by $0<\mathrm{E}<(\mathrm{k}+1) \mathrm{E}_{\max }, 0<\varepsilon<\mathrm{kE}_{\max }$, and $0<E-\varepsilon<E_{\max }$, is represented by the shaded parallelogram in the figure.

Therefore, we can recast the double integral in the form

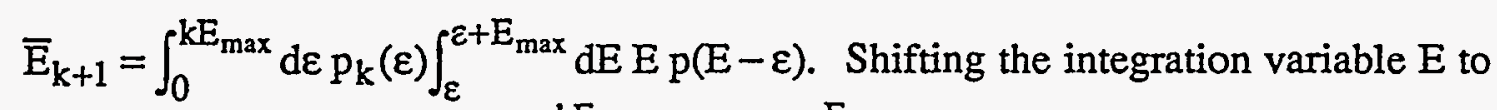
$E^{\prime}=E-\varepsilon$, we obtain $\bar{E}_{k+1}=\int_{0}^{k E_{\max }} d \varepsilon p_{k}(\varepsilon) \int_{0}^{E_{\max }} \mathrm{dE}^{\prime}\left(E^{\prime}+\varepsilon\right) p(\varepsilon)$, which leads to Eq. (7).

Fig. 2. Total cross section for inelastic collisions of a fast charged particle with atoms, plotted against atomic number $Z$ (reproduced with permission from Inokuti et al. [13]). The broken line shows the result based on the nonrelativistic expression, Eq. (29). The solid line shows the result of relativistic effects and is therefore more realistic. 


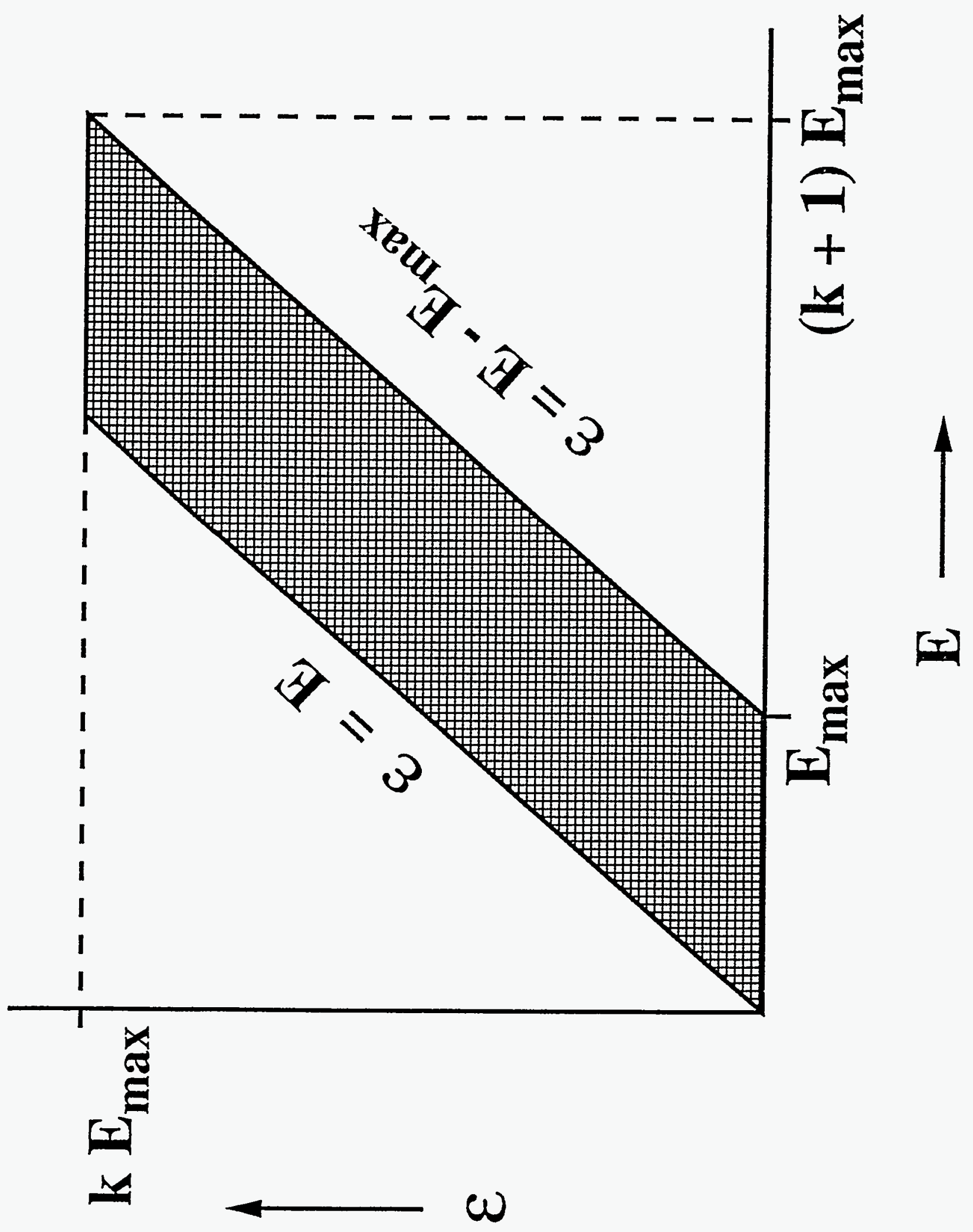




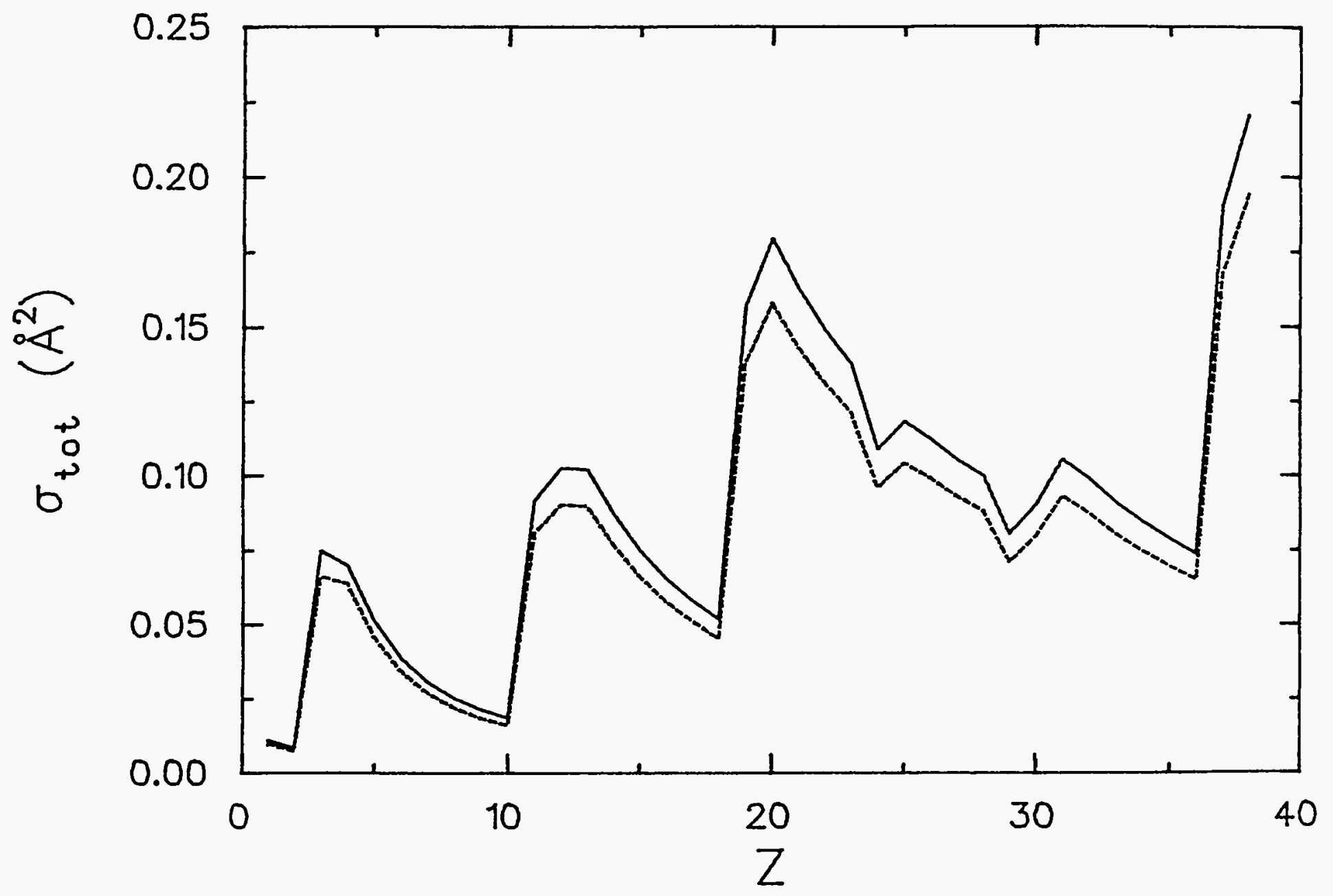

Fig. 2 of Inokuti for NIII B 